\title{
UBIQUOX, plataforma de investigación de mercado en línea
}

\author{
UBIQUOX, online market research platform
}

UBIQUOX, plataforma de pesquisa de mercado online.

\section{ARTÍCULO ORIGINAL}

\section{Alan Antonio Hernández López}

\author{
NET CONSULTORES S.A.C. ciudad de Lima, Perú. \\ ahernandez@net-consultores.com \\ https://orcid.org/0000-0003-4965-6150.
}

Recibido 05 de Febrero 2021 | Arbitrado y aceptado 19 de Abril 2021 | Publicado en 28 Julio 2021

\section{RESUMEN}

En el año 2019, la empresa NET CONSULTORES S.A.C., con la finalidad de expandir su mercado de productos relacionados a desarrollo de software, implementación de soluciones informáticas y consultoría, decide realizar la elaboración de una investigación de mercado (QuestionPro, 2020) y foros para conocer las necesidades de las diversas empresas, a través de entrevistas a sus principales representantes, gerentes o encargados del negocio.

Después de una ardua búsqueda para seleccionar la empresa consultora de marketing, en el mes de julio de 2018 se encargó el estudio. Aquel, demoró aproximadamente un mes y medio en la entrega de los resultados, lo cual es una duración inaceptable para una investigación que requiere la evaluación del mercado en un instante de tiempo en el cual los escenarios son tan cambiantes, sobre todo ahora con la revolución y transformación digital.

A partir de esa experiencia, NET CONSULTORES decidió invertir tiempo y recursos para conceptualizar una herramienta que acelerará este proceso con un servicio acotado y dirigido.

Después de tres meses de definiciones y conversaciones con empresas de sectores multiempresariales, se estableció que el alcance inicial de la solución debería ser la automatización de estudios de muestreo.

Así, nace UBIQUOX (UBIQUOX, 2021), la primera plataforma de investigación de mercado en línea, para que, con un precio adecuado y de forma rápida, las empresas puedan realizar averiguaciones de su entorno y facilitar la toma de decisiones para dirigir su negocio hacia las necesidades de sus clientes, garantizando un retorno de su inversión y potencialidades de crecimiento diverso.

Palabras clave: Investigación de mercado, en línea.

\section{ABSTRACT}

In 2019, the company NET CONSULTORES $\mathrm{SAC}$, in order to expand its market of products related to software development, implementation of computer solutions and consulting, decides to carry out a market research (QuestionPro, 2020) and forums for know the needs of the various companies, through interviews with their main

representatives, managers or those in charge of the business.

After an arduous search to select the marketing consulting company, the study was

commissioned in July 2018. That one took approximately a month and a half to deliver the results, which is an unacceptable duration for an investigation that requires the evaluation of the market in a moment of time in which the scenarios are so changing, especially now with the revolution. and digital transformation. Based on that experience, NET CONSULTORES decided to invest time and resources to conceptualize a tool that will accelerate this process with a limited and targeted service.

After three months of definitions and conversations with companies in multicompany sectors, it was established that the initial scope of the solution should be the automation of sampling studies. Thus, UBIQUOX (UBIQUOX, 2021) was born, the first online market research platform, so that, with an appropriate price and quickly, companies can make inquiries of their environment and facilitate decision-making to direct their business towards the needs of its clients, guaranteeing a return on investment and diverse growth potential.

Keywords: Market Research, Online

\section{RESUMO}

Em 2019, a empresa NET CONSULTORES SAC, com o objetivo de expandir o seu mercado de produtos relacionados com o desenvolvimento de software, implementação de soluções informáticas e consultoria, decide realizar uma pesquisa de mercado (QuestionPro, 2020) e fóruns para conhecer as necessidades dos diversos empresas, por meio de entrevistas com seus principais representantes, gestores ou responsáveis pelo negócio.

Depois de uma árdua busca para selecionar a empresa de consultoria de marketing, o estudo foi encomendado em julho de 2018. Aquela demorou cerca de um mês e meio a entregar os resultados, duração inaceitável para uma investigação que exige uma avaliação do mercado num momento em que os cenários estão tão mudando, principalmente agora com a revolução. E digital transformação.

Com base nessa experiência, a NET CONSULTORES decidiu investir tempo e recursos para conceituar uma ferramenta que acelere esse processo com um atendimento limitado e direcionado.

Após três meses de definições e conversas com empresas de setores multiempresas, ficou estabelecido que o escopo inicial da solução deveria ser a automação de estudos de amostragem.

Nasceu assim a UBIQUOX (UBIQUOX, 2021), a primeira plataforma de pesquisa de mercado online, para que, com um preço adequado e com rapidez, as empresas possam realizar inquéritos sobre o seu ambiente e facilitar a tomada de decisões para direccionar os seus negócios às necessidades dos seus clientes, garantindo um retorno do investimento e um potencial de crescimento diversificado.

Palavras-chave: Pesquisa de Mercado, Online 


\section{INTRODUCCIÓN}

Actualmente, los estudios para la investigación de monitoreo de precios y medición de calidad de servicios de atención al cliente son lentos y costosos (demoran como mínimo un mes para su desarrollo). Adicionalmente a ello, la generación de encuestas en línea hacia un público directo para efectos de lanzamiento de productos, estrategias de comercialización y publicidad no se realizan porque no existe una comunidad con segmentos identificados (edad, sexo, ubicación geográfica, etc..) donde se pueda "testear" rápidamente una idea o innovación. También, en este momento no existe una herramienta de rápido monitoreo de cumplimiento de protocolos de salud para disminuir el riesgo de contagio de la COVID - 19.

Estos escenarios anteriormente descritos ocasionan que emprendedores, Micro y pequeñas empresas (MYPES) y Pequeñas y Medianas Empresas (PYMES) no accedan a este tipo de estudios que permite testear un producto, idea o medir la calidad de servicio al cliente.

En referencia a monitoreo de precios, existe interés internacional de empresas latinoamericanas y de Estados Unidos (EEUU) en este tipo de servicio, para investigar sobre el valor de un determinado producto en una localización y evaluar su ingreso al mercado nacional. Actualmente este servicio no es conocido y se identificó su necesidad a partir de reuniones con empresas colombianas dirigidas por PROCOLOMBIA, entidad gubernamental de Colombia a cargo de promover las exportaciones de este país.

UBIQUOX se crea para cubrir esta necesidad del mercado, ofrece la posibilidad de brindar este tipo de servicio de investigación en línea a través de la automatización de los procesos. NET CONSULTORES S.A.C. empresa reconocida con más de 10 de años de experiencia profesional en el mercado de soluciones informáticas, decide plantear el desarrollo de esta plataforma estimando los recursos necesarios para la obtención del producto.

En el mes de abril del 2020, NET CONSULTORES decide participar en el programa de Proyectos de Innovación Empresarial, categoría 1, individual INNOVATE PERÚ (Ministerio de la Producción, 2021), con código de proyecto: PIEC-6-P-244-071-20 presentando la solución bajo la denominación: "UBIQUOX - PLATAFORMA PARA INVESTIGACIÓN DE MERCADO EN LÍNEA, bajo los siguientes lineamientos: 
Área de Innovación: TECNOLOGÍA DE INFORMACIÓN Y DEL CONOCIMIENTO.

Actividad Económica en la que se aplicará la innovación: INDUSTRIAS DE LA INFORMACIÓN Y DEL CONOCIMIENTO.

Localización del proyecto: LIMA, PERÚ.

Duración del proyecto: 10 meses.

Presupuesto por entidades aportantes:

- $\quad$ NET CONSULTORES S.A.C. $\quad$ S/. 184, 632.00

- INNOVATE PERÚ: S/. $\quad$ S66,548.00

Después de superar el proceso de evaluación del programa INNOVATE PERÚ (ahora llamado ProInnóvate), se firma el contrato 219-INNOVATEPERU-PIEC1-2020 asignando a NET CONSULTORES S.A.C. como entidad ejecutora para el desarrollo de la plataforma UBIQUOX, estableciendo la fecha de inicio del proyecto, el dos de noviembre de 2020 con los siguientes hitos de proyecto (4) y sus correspondientes entregables:

Tabla 1: Cuadro de hitos del proyecto

\begin{tabular}{|c|c|c|c|}
\hline Hitos & $\begin{array}{l}\text { Fecha Inicio de } \\
\underline{\text { Hito }}\end{array}$ & $\begin{array}{l}\text { Fecha Fin de } \\
\underline{\text { Hito }}\end{array}$ & $\underline{\text { Indicadores del Hito }}$ \\
\hline 1 . & 02-nov-2020 & 01 -ene-2021 & $\begin{array}{l}\text { 1. Informe de Sustento Técnico } \\
\text { de componentes } \\
\text { considerados para la } \\
\text { investigación de mercado. } \\
\text { 2. Informe de identidad } \\
\text { publicitaria, incluyendo } \\
\text { Manual de Marca del } \\
\text { producto UBIQUOX y } \\
\text { Medición del Grado de } \\
\text { Aceptación de la Plataforma } \\
\text { UBIQUOX. }\end{array}$ \\
\hline 2. & 02-ene-2021 & $01-a b r-2021$ & $\begin{array}{l}\text { 1. Informe de la realización del } \\
\text { portal web incluyendo: }\end{array}$ \\
\hline
\end{tabular}




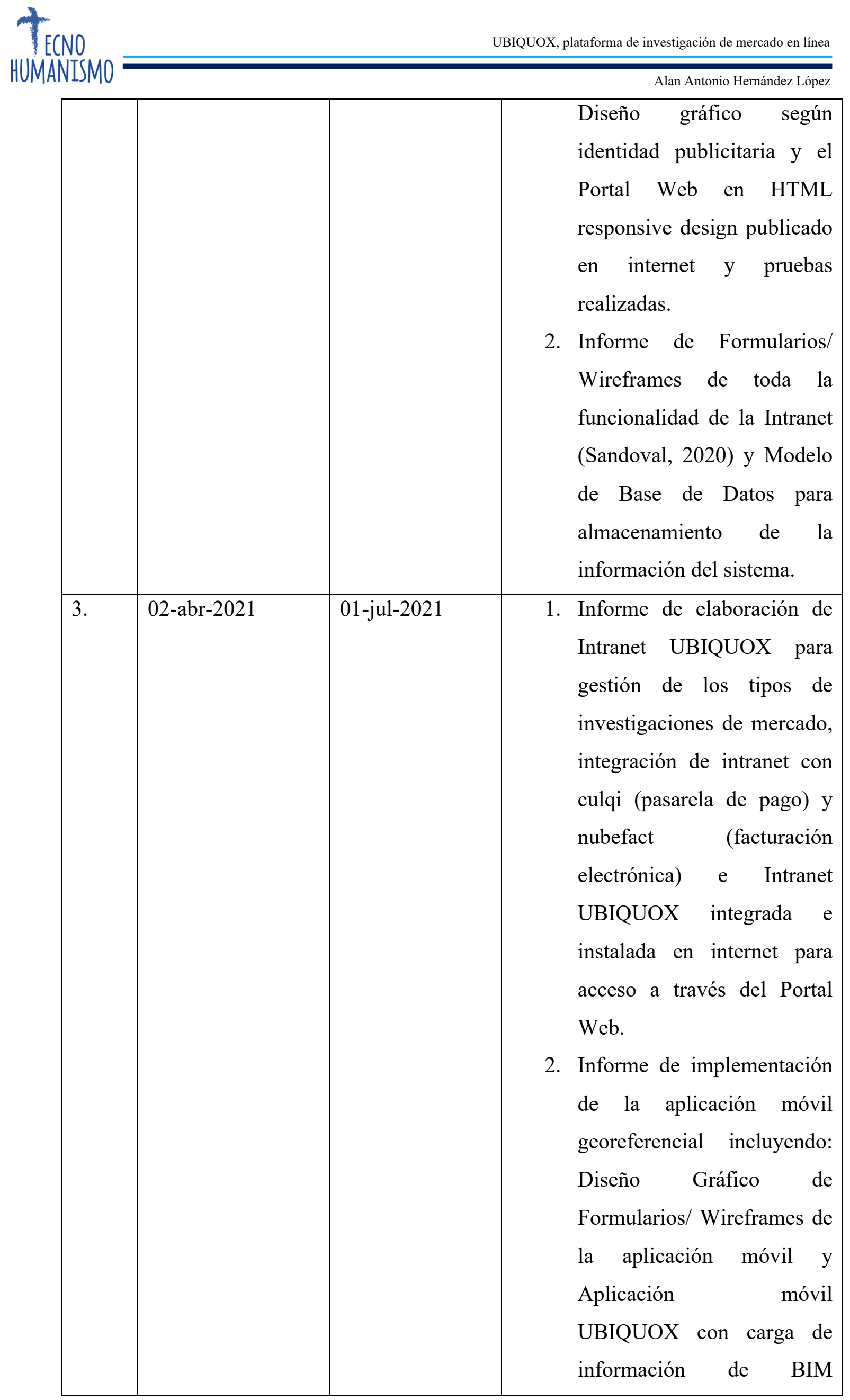




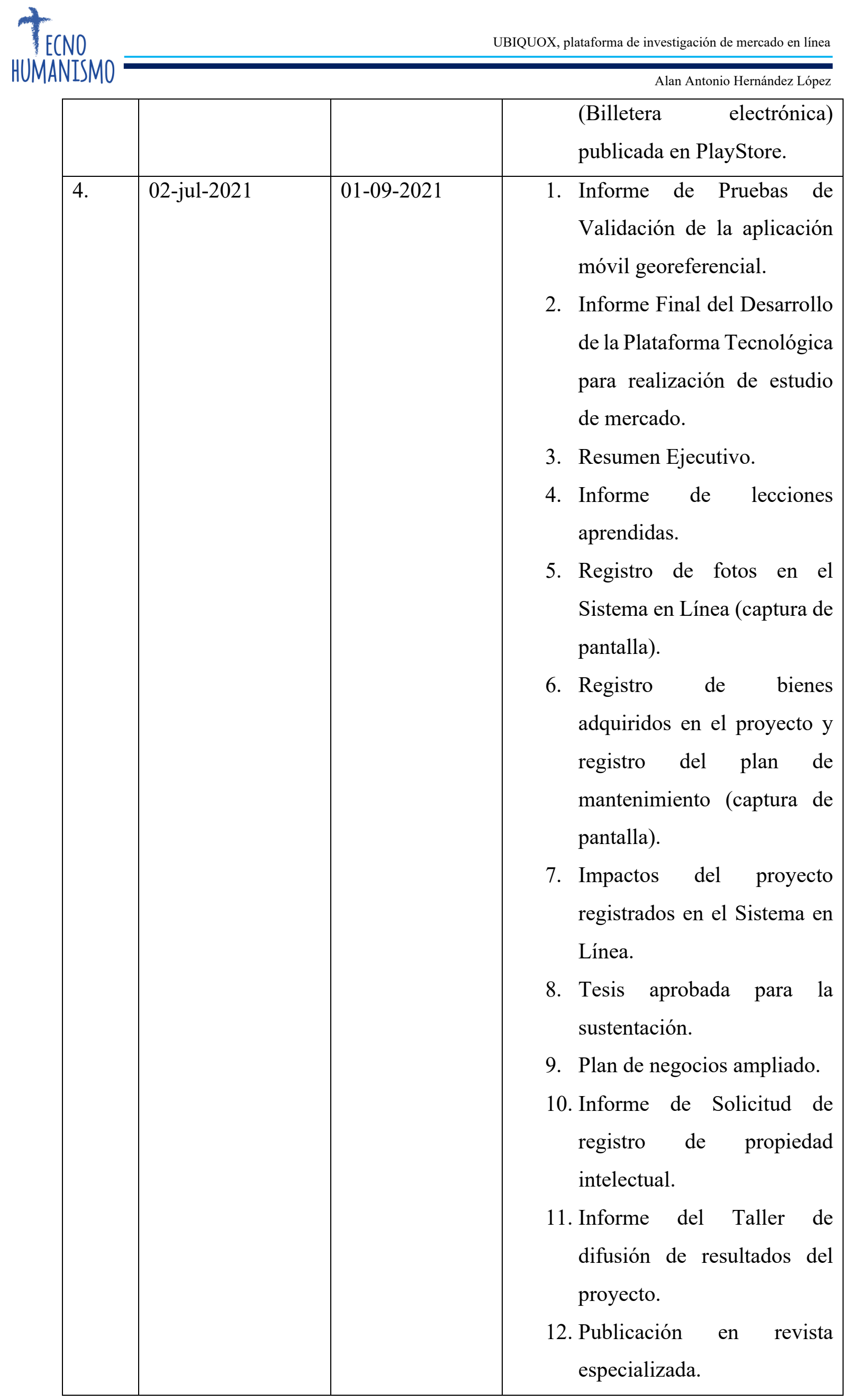




\section{DESARROLLO}

Para este punto, nos centraremos en el alcance tecnológico de UBIQUOX PLATAFORMA DE INVESTIGACIÓN DE MERCADO EN LÍNEA.

Los componentes de la solución informática son los siguientes:

- Portal Web (Rodríguez, 2012), para la publicación de la información relacionada a la plataforma, registro de personas naturales y jurídicas e ingreso a la intranet de clientes.

- Intranet para clientes, con la finalidad de realizar la configuración de las investigaciones de mercado según la tipología planteada (monitoreo de precios, encuesta o monitoreo de servicios), realizar el pago según el cálculo automático del alcance en integración con pasarela de pago y facturación electrónica, así como también la visualización en línea de los resultados del estudio de mercado.

- Aplicación móvil (Herazo, 2019), aplicación georeferencial para el registro de las personas naturales que accederán a las investigaciones en línea, solicitadas por la intranet de clientes, para la recopilación de los datos y consolidación de los mismos en los reportes en línea.

Como metodología de desarrollo de software, NET CONSULTORES planteó y ejecutó las siguientes fases:

- Fase de Inicio, etapa en la cual se realizaron las siguientes actividades:

- Análisis de procesos para la identificación detallada de los requerimientos a desarrollar, obteniendo el INVENTARIO DE REQUERIMIENTOS FUNCIONALES.

- Análisis técnico para la definición de la arquitectura de software obteniendo el INVENTARIO DE REQUERIMIENTOS NO FUNCIONALES.

- Diseño de formularios/ Wireframes/ prototipos de pantalla detallados de los procesos identificados para realizar el despliegue de desarrollo de software (PORTAL WEB, INTRANET DE CLIENTES Y APP MÓVIL EN ANDROID).

- Fase de Elaboración, etapa en la cual se realizaron las siguientes actividades:

○ Diseño del Modelo Entidad Relación.

- Elaboración del Diccionario de Datos. 
- Elaboración del Documento de Arquitectura de Software.

- Implementación del prototipo base de arquitectura de software para incorporación de las funcionalidades requeridas.

- Fase de Construcción, etapa en la cual se realizaron las siguientes actividades:

- Implementación del Portal Web UBIQUOX.

- Desarrollo de los Módulos del Sistema (Intranet de Clientes, Módulo de Administración y Aplicación Móvil en Android).

- Pruebas iniciales de los módulos de la Plataforma UBIQUOX.

- Fase de Validación, etapa en la cual se realizaron las siguientes actividades:

- Pruebas finales.

- Integración de todos los componentes de la plataforma UBIQUOX.

- Carga de datos iniciales.

○ Pruebas de vulnerabilidad y seguridad informática.

- Fase de Transición, etapa en la cual se realizaron las siguientes actividades:

○ Puesta en producción.

- Capacitación a usuarios.

- Preparación de entregables y contingencia de datos.

- Cierre del proyecto.

Como es sabido, esta metodología es estándar y básica para el desarrollo de software y es llamada también metodología de cascada (Digital Guide IONOS, 2020).

Sin embargo, NET CONSULTORES determinó e implementó la metodología ágil llamada XP - Xtreme Programing (Sinnaps, 2020) para rápidamente identificar en dos iteraciones (la primera al tercer mes de desarrollo y la segunda al quinto mes) los cambios, adecuando la plataforma para mayor robustez. Así, disminuyó el riesgo en la generación del producto final y procedió a testear la solución en dos empresas afines para validar la funcionalidad completa de la intranet de clientes en integración con la aplicación móvil en Android, obteniendo resultados satisfactorios.

Debido a la COVID-19, el desarrollo del proyecto en su totalidad, ha sido ejecutado bajo la modalidad de teletrabajo, incluso en coordinación con las empresas que brindaron los servicios de terceros, 
Toda la inversión planteada ha sido dirigida hacia la obtención de los hitos del proyecto y sus correspondientes indicadores, todo ello con la finalidad de garantizar su ejecución con sostenibilidad y viabilidad ante el programa INNOVATE PERÚ.

\section{CONCLUSIONES}

A partir de la finalización del proyecto y la obtención del producto UBIQUOX, PLATAFORMA DE INVESTIGACIÓN DE MERCADO EN LÍNEA con el cofinanciamiento del programa INNOVATE PERÚ (ahora llamado ProInnóvate), se presentan las siguientes conclusiones:

- La herramienta brindará los servicios automatizados de Mistery Shopping (cliente misterioso), encuestas en línea, monitoreo de precios de productos para identificar la competencia e introducción en el mercado y medición de calidad de servicio (pre y post).

- La duración del proyecto planificado y ejecutado, ha sido de 10 meses. Con fecha de inicio, el 02 de noviembre de 2020 y fecha de fin el 01 de setiembre de 2021.

- La inversión del programa INNOVATE PERÚ ha sido un factor crítico de éxito para la generación de la plataforma y el cumplimiento de los indicadores por hitos. A partir de los avances presentados conjuntamente con los desembolsos en los momentos apropiados, han permitido que el proyecto fluya con naturalidad en su ejecución.

- La experiencia de NET CONSULTORES y del equipo de trabajo del proyecto, ha permitido identificar con anticipación, los cambios necesarios a implementar en la plataforma UBIQUOX.

- Para la comercialización de la plataforma, se ha determinado un modelo de negocio tipo SaaS (Software as a Service), UBIQUOX se encuentra instalado en servidores virtuales y se puede acceder a través de la dirección URL: $\underline{\text { ubiquox.com }}$

- UBIQUOX estará dirigido a emprendedores, MYPES y PYMES y ha sido construida con la finalidad de "democratizar" el proceso de investigación de mercado tipo población muestreo.

- UBIQUOX permitirá que personas naturales independientes se favorezcan, obteniendo ingresos por participar en las investigaciones en línea requeridas por los clientes, mediante el uso de la aplicación móvil. Para ello, en la siguiente etapa de lanzamiento de la plataforma, se generará una comunidad virtual a través de las redes sociales y la estrategia de marketing digital para la captación de las 
personas naturales que descarguen la app, así como los clientes interesados en realizar los estudios que la herramienta brinda.

- A la fecha, no se ha identificado competencia directa con UBIQUOX. De manera indirecta, se encuentran las empresas de marketing que ofrecen este tipo de servicio de manera tradicional. De esta manera, existe la posibilidad de realizar alianzas estratégicas con ellas para posicionar la plataforma.

- El canal de distribución es online, es decir, todo el proceso se encuentra automatizado: Desde la solicitud de la generación del estudio, el pago en línea, la disponibilidad de abrir la investigación, la recopilación de datos por las personas naturales y el análisis en línea de los resultados obtenidos.

- Como conclusión final se menciona que el producto obtenido es la versión 1.0, existiendo la posibilidad de continuar con su mejora, a partir del lanzamiento y posicionamiento en el mercado. Para lograr este objetivo, NET CONSULTORES participará en la segunda categoría de proyectos llamada Validación de la Innovación del Programa Nacional de Innovación para la Competitividad y Productividad, INNOVATE PERÚ (ahora 1lamado ProInnóvate).

\section{Bibliografía}

1. QuestionPro. (2020). Introducción a la Investigación de Mercados. Recuperado de https://www.questionpro.com/es/investigacion-de-mercados.html

2. UBIQUOX (2020). Recuperado de ubiquox.com

3. Ministerio de la Producción (2020).Programa ProInnóvate. Recuperado de www.innovateperu.gob.pe

4. Rodríguez, D. (2012). Portales web empresariales. ¿Qué son y cuáles son sus funciones? Recuperado de https://www.gestiopolis.com/portales-webempresariales-que-son-y-cuales-son-sus-funciones/

5. Sandoval, L. (2020). ¿Qué es intranet? y ¿Para qué sirve? Recuperado de https://ventanainformatica.com/c-informatica/que-es-la-intranet/

6. Herazo, L (2019). ¿Qué es una aplicación móvil? Recuperado de https://anincubator.com/que-es-una-aplicacion-movil/

7. Digital Guide IONOS (2020). El modelo en cascada: desarrollo secuencial de software. Recuperado de https://www.ionos.es/digitalguide/paginasweb/desarrollo-web/el-modelo-en-cascada/

8. Sinnaps. (2020). Metodología XP o Programación Extrema. 


\section{ISOTIPO UBIQUOX}

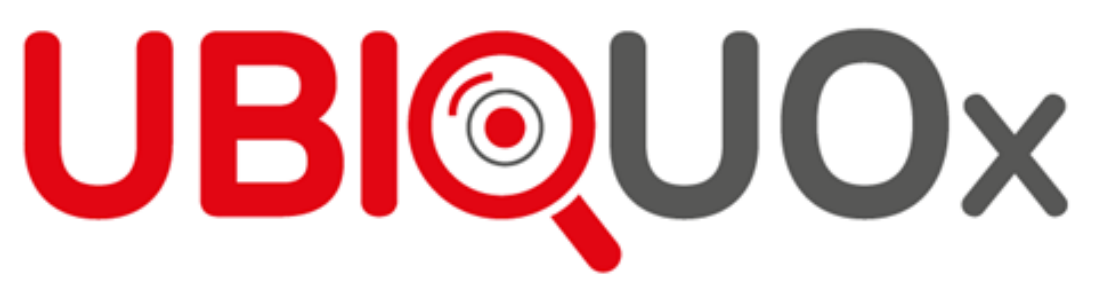

